\title{
Voix ancestrales et Écologie profonde : le chant comme convergence entre paysage, politique et émotion en milieu montagnard
}

\section{Ancient Voices and Deep Ecology: Song as a convergence between landscape, politics and emotion in mountain society}

\author{
Jennifer Bonn Lombard ${ }^{1}$ \\ ${ }^{1}$ créatrice sonore, doctorante en Études audiovisuelles au laboratoire LARA-SEPPIA, Université de Toulouse Jean- \\ Jaurès
}

RÉSUMÉ. Le chant active à la fois des fonctions corporelles, des sièges émotionnels et une production intellectuelle. Les trois entrent par le chant en échange avec l'espace dans lequel se trouve le chanteur. En montagne pour les montagnards des communautés d'altitude, cet espace correspond à une source majeure de nourriture pour le corps physique, d'expériences pour l'affect et d'inspiration pour l'intellect. Le chant est à la fois un hommage, une conversation, et une négociation avec cette Nature qui peut se montrer généreuse comme avare, bienveillante comme menaçante.

À travers des exemples tirés de recherches menées dans les Pyrénées françaises et dans les Andes argentines, cet essai permettra de découvrir comment la fonction et le fonctionnement du chant ancestral forgent, maintiennent et soignent des relations complexes et essentielles avec le monde naturel, et comment la perspective environnementale qu'ouvrent ces relations s'approchent d'une réflexion contemporaine sur l'écologie telle que la pratique des chercheurs comme Arne Naess, Isabelle Stengers, Donna Haraway ou Brian Massumi. Une relation au lieu qui est un engagement profond fusionnant émotion et politique, corps et espace.

ABSTRACT. Song simultaneously activates body functions, emotional centers and the intellect. All three participate in the exchange that the singer initiates with his or her environment. In the mountains, for the communities who live there, this environment is a major source of sustenance for the physical body, of experiences for generating emotional response, and of inspiration for the intellect. Song is an ode, a conversation, but also a negociation with Nature, and the natural environment can be generous or miserly, benevolent or threatening.

Through examples taken from research in both the French Pyrenees and the Argentinian Andes, this essay will allow us to discover how the functions and effects of ancestral song forge, maintain and care for complex, essential relationships with the natural environment, and how the environmental perspectives that these relationships open resonate with contemporary thinking on ecology as it is practiced by researchers such as Arne Naess, Isabelle Stengers, Donna Haraway or Brian Massumi. A relation to place that which is a deep commitment that merges emotion and politics, body and space.

MOTS-CLÉS. Chant, montagne, émotion, paysage, politique, Pyrénées, Andes.

KEYWORDS. Song, mountain, emotion, landscape, politics, Pyrenees, Andes.

Le chant active à la fois des fonctions corporelles, des sièges émotionnels et une production intellectuelle. Les trois entrent par le chant en échange avec l'espace dans lequel se trouve le chanteur. En montagne pour les montagnards des communautés d'altitude, cet espace correspond à une source majeure de nourriture pour le corps physique, d'expériences pour l'affect et d'inspiration pour l'intellect. Le chant est à la fois un hommage, une conversation, et une négociation avec une Nature qui peut se montrer généreuse comme avare, bienveillante comme menaçante.

Les communautés de montagne de la Haute-Soule située en Pays basque français, et celles des provinces de Salta et Jujuy dans le nord-ouest argentin, présentent à la fois des particularités et des similarités concernant le lien créé par le chant par ces populations avec le milieu naturel. Le chant 
dans ces communautés ne se réduit pas à symboliser ce lien, mais participe à le forger, à le maintenir et à le soigner ${ }^{1}$. En cela le chant pose activement les bases d'une forme d'écologie qui résonne avec certains courants écologiques actuels.

Pour comprendre cet échange à multiples entrées entre un corps et un espace, entre un individu et son lieu, il faut considérer dans un premier temps séparément les vecteurs physiques, émotionnels, et intellectuels empruntés par le chanteur.

Dans le chant, il y a un engagement physique qui passe par la technique même du chant. Là déjà, une interaction très concrète opère entre le chanteur et l'espace dans lequel il se trouve. Le corps est ancré, debout, stable et en contact actif avec le sol. L'endroit où se tient le corps est un endroit choisi. En montagne, ce choix peut correspondre à une vue singulière ou à une acoustique particulière. Dans tous les cas, cela implique une perception de l'espace par le corps, et l'insertion de ce corps dans l'espace. Ensuite, le chanteur engage sa respiration. Dans le chant ancestral du nordouest argentin qui s'appelle le canto con caja, cette respiration est pensée comme une double circulation qui part du plexus solaire, dont une boucle descend vers le bas pour puiser dans le sol et dont une autre part vers le haut pour puiser dans le ciel. Cet échange d'air, précisément parce que le chanteur y porte son attention, est une mise en valeur d'un lien physique avec l'environnement. L'air qui circule est partagé par le chanteur et par la montagne. La voix du chanteur s'engage dans cet espace, et le chant n'est pas seulement l'émission de la voix, mais aussi l'écoute simultanée qui l'accompagne et qui le modifie. La montagne renvoie au chanteur sa réponse, sous forme de résonance, d'écho, ou d'absorption, et le chanteur module son chant pour mieux jouer avec son amphithéâtre naturel. Dans les montagnes du Pays basque, il existe un chant sans parole nommé basa ahaide, où la voix du chanteur initie par la montée des notes une ascension dans les contours et les accidents du relief, en suivant par son imaginaire la trace des thermiques dessinée par le mouvement des oiseaux qui s'en servent pour atteindre les sommets. Les surfaces qui composent le milieu sont un tremplin pour la voix, permettant un jeu à la fois technique et esthétique. Dans les Andes du nord-ouest argentin, chaque communauté chante sur une mélodie qui lui est propre, et qui correspond à la forme de son paysage : les communautés vivant dans les gorges et canyons ont un chant plus étiré, laissant de la place aux renvois impressionnants des parois rocheuses, et les communautés des espaces ouverts de l'altiplano ont un chant plus rythmé et continu, pour maintenir la présence du son face au vide qui l'engloutit.

Ces chants sont une forme de communion ou de symbiose avec la montagne. Le corps s'étend par la voix dans le paysage, faisant de ce paysage une extension du corps, mais le corps se dissout aussi dans le paysage, il devient en quelque sorte ce paysage. Cela suscite chez le chanteur, comme chez d'éventuels auditeurs, de l'émotion. Ce que le chanteur puise en soi pendant le chant correspond à son état d'âme, et que ce soit de la joie ou de la tristesse, c'est cet état très intime qu'il partage avec la montagne, et que la montagne par sa réponse va alimenter à son tour. Très souvent joie et tristesse se mélangent dans la beauté du chant, et le chant travaille sur une intensification de cet état émotionnel. Dans le basa ahaide basque par exemple, l'intensité du chant monte avec la montée dans les aigus par une voix qui ne quitte pas la voix de poitrine pour entrer dans la voix de tête ; la voix est donc poussée dans un son très concentré et puissant dont on a l'impression qu'il finit par dépasser en quelque sorte le corps du chanteur, par exploser dans l'atmosphère. Le chant frôle le cri, touche des sonorités perçantes rappelant la voix des rapaces et la résonance du minéral. En cela le basa ahaide

\footnotetext{
Toutes les informations concernant les chants des Pyrénées et des Andes qui sont exposées dans cet essai proviennent d'une thèse en cours de rédaction par l'auteur : BONN Jennifer, Chant Terrestre, la voix comme créatrice de lien en milieu montagnard.
} 
évoque à la fois la tristesse d'une lamentation et la joie d'un cri de liberté. Souvent, les basa ahaide finissent sur une non-fin, dans le sens où par le phrasé du chant on sent intuitivement qu'il devrait continuer, qu'il invite à continuer. Et peut-être continue-t-il encore, loin du corps du chanteur, sa voix ayant rejoint les oiseaux.

Dans le canto con caja argentin, l'émotion a un double vecteur : la mélodie et les paroles. Les mots, qui prennent la forme d'un poème à quatre strophes appelé copla, exprime les émotions du chanteur, ses joies et ses plaintes, ses impressions et ses espoirs. Par le poème, le chant va stimuler l'intellect du chanteur, permettant une analyse et une traduction en mots de son environnement ainsi que de son mode de vie au sein de cet environnement. Les paroles des coplas dans le canto con caja sont presque toujours liées à un environnement naturel, le chanteur se plaçant d'abord dans son lieu en le décrivant. La description qu'il fait du lieu est ce qui va refléter sa situation personnelle et même sa personnalité, ce qui va permettre de dire si la vie est faste ou dure, si la Nature est généreuse ou avare. Mais cette description permet aussi de dire si ce lieu est menacé, par quoi ou par qui, et parfois d'exprimer le chagrin du chanteur d'avoir été arraché à ce lieu. Par ces glissements qui passent souvent par des métaphores ou des allusions à mi-mots, le chant devient plus ouvertement politique, bien que l'on peut reconnaitre que tout engagement envers un lieu en lien avec la vie d'une communauté est déjà un engagement ayant des répercussions politiques. Voici un exemple parmi les centaines de coplas du répertoire andin :

Esta zorra voy llegando de lejos.

Cette renarde-ci vient de loin.

Vengo, de lejos vengo,

Je viens, de loin je viens,

porque temprano no fue a buscar

parce que je ne suis pas partie tôt

la suerte, que alla no tengo. ${ }^{2}$

chercher la fortune que je n'ai pas là-bas.

Dans ce copla, la chanteuse s'identifie à la renarde, sauvage et solitaire, qui erre dans son paysage natal désertique. Elle n'est pas partie jeune de chez elle pour chercher fortune (sous-entendant que beaucoup d'autres l'ont fait), et témoigne de la dureté de la vie dans son village, où ce n'est pas une chance de vivre. Mais malgré l'hostilité de son environnement, la chanteuse exprime aussi sa fierté d'y appartenir : la renarde est endurante, futée, et capable de survivre dans des milieux hostiles.

Quand on considère que les villages andins ont été vidés par l'exode vers les villes et qu'une grande partie de la population a été exploitée, et continue souvent de l'être, dans les industries urbaines qui font miroiter une meilleure qualité de vie aux ouvriers, ce chant en apparence très simple révèle des strates profondes avec des implications clairement politiques et sociales. Le prix à payer pour la liberté du renard est de devoir vivre avec la difficulté, mais cette liberté est plus importante que le petit confort auquel aspire l'ouvrier.

Le chant opère donc une triple activation : physique, émotionnelle et intellectuelle, qui relie le chanteur au lieu dans lequel il se trouve, ce qui dans le chant ancestral correspond à son territoire, à sa terre, à l'environnement de son quotidien. Il y a là une forme d'écologie, qu'il s'agit maintenant d'explorer plus en détail, en essayant de voir en quoi elle se rapproche de certains courants contemporains, et donc ce qu'elle propose comme réflexion vis-à-vis de l'actualité écologique. 
L'écologie profonde, ou Deep Ecology, est un mouvement écologique qui a pris racine aux EtatsUnis dans les années soixante-dix, mais que le grand public découvre à peine en France avec la traduction relativement récente des travaux d'Arne Naess ${ }^{3}$. Naess est un philosophe et environnementaliste norvégien qui s'est inspiré pour formuler sa notion d'écologie profonde, ainsi que l'écosophie qui l'accompagne, des écrits de Rachel Carson, notamment son texte fondateur Silent Spring, mais aussi ceux de Henry David Thoreau, de John Muir et d'Aldo Leopold, entre autres. Le travail de Naess a servi à fonder le mouvement de Deep Ecology, porté ensuite principalement aux Etats-Unis par des activistes, penseurs et artistes tel que Gary Snyder, Wendell Berry ou Stephan Harding, pour ne citer que quelques uns.

Naess pose déjà les bases d'une pensée complexe du milieu naturel, où tous les éléments, l'homme inclus, sont en interaction et en interdépendance permanentes, et où chaque être vivant est valorisé indépendamment de son utilité pour l'homme. Cela a été largement repris par les mouvements écologiques actuels. Mais Naess propose autre chose qui est particulièrement intéressant dans le cadre de cet essai, et qui est le lien intrinsèque entre l'expérience émotionnelle, l'établissement de valeurs et l'engagement politique. Il explique comment ces trois choses, quand elles sont appliquées à un écosystème, vont permettre d'établir et de renforcer les convictions politiques d'une écologie profonde.

L'expérience du chant, que ce soit le basa ahaide basque ou le canto con caja argentin, suscite une émotion en lien avec la Nature, à laquelle le chant est dédié. Cette émotion participe à maintenir un lien fort entre le chanteur et sa terre, elle renforce son sentiment d'appartenance au lieu. Selon Naess, «le processus selon lequel une émotion se transforme en une évaluation n'est pas tant un déplacement effectif qu'un déplacement d'accent fondé sur la reconnaissance du rôle crucial que jouent les sentiments dans l'élaboration des diverses visions du monde ». Les émotions qui se produisent spontanément en relation avec un milieu naturel, et que le chant facilite et amplifie, se traduisent, grâce à la réflexion, grâce à l'interprétation par l'intellect, en des évaluations qualitatives qui, petit à petit, définissent les critères d'une qualité de vie vécue ou souhaitée par un individu au sein de son milieu. L'engagement politique surgit de la volonté de maintenir, de protéger ou de rétablir cette qualité de vie et donc ce milieu naturel.

Nous pouvons voir le cheminement proposé par Naess dans les fonctions et les paroles du canto con caja. Le sentiment d'appartenance à un milieu naturel donne naissance à des coplas qui décrivent cet environnement et qui expriment la fierté de l'homme d'y appartenir. Mais il existe aussi des coplas qui défendent ouvertement ce milieu naturel et le mode de vie que mènent ses habitants face aux menaces extérieures telles que l'expropriation, l'exploitation des hommes et des ressources, et l'oppression de la culture traditionnelle, culture qui est très en lien avec le milieu naturel. En voici un exemple parmi beaucoup d'autres :

Esta copla hoy le canta

el viento à la cordillera :

« con la mina a cielo abierto

nos muere el agua y la tierra $»^{4}$
Aujourd'hui, cette copla est chantée

par le vent à la cordillère :

« avec la mine à ciel ouvert

ils nous font mourir l'eau et la terre » 
Le chanteur transmet le chant que lui propose (que lui inspire) son milieu naturel, inquiet des conséquences fatales de l'industrie minière sur ce qui le compose, sur le corps du milieu. Ainsi on projette une corporalité et un sentiment de souffrance sur la Nature qui permet au chanteur et aux auditeurs de s'identifier au milieu naturel tout en leur enjoignant de le protéger. L'enchaînement émotion - valeur - engagement politique fait ici intrinsèquement partie de la pratique du chant.

Au Pays basque, le basa ahaide n'ayant pas de paroles, l'enchaînement se fait dans ce cas par un autre cheminement. C'est d'abord l'esthétique sonore, la beauté du chant, ainsi que l'esthétique visuelle, la beauté de l'environnement dans lequel il est chanté, qui suscitent l'émotion. Ensuite c'est l'association de cette pratique à la tradition basque et donc sa participation à la définition de ce qui serait "profondément basque" (et même profondément soulétin, puisque le basa ahaide est originaire de la partie la plus montagneuse du Pays basque français) qui permet de créer les valeurs de la communauté. Ces valeurs sont ensuite défendues face à ce qui pourrait les mettre en danger, traduisant un engagement politique.

Le basa ahaide et la copla sont des chants de berger, et donc intimement liés au mode socioéconomique du pastoralisme en montagne. Dans les Pyrénées comme dans les Andes, la réussite dépend à la fois de la coopération entre les membres de la communauté et des conditions propices $\mathrm{du}$ milieu. Le chant travaille à favoriser ces deux aspects, par la convivialité, par l'attention au contexte, par la valorisation des ressources ainsi que de la tradition, et par la mise en garde contre des dangers éventuels.

Mais cela ne veut pas dire que la Nature défendue par ces communautés est infiniment parfaite, bonne et généreuse. Pour ces peuples millénaires, la question n'est pas si simple. La Nature peut être avare, dure, hostile, destructrice, meurtrière. Si on veut qu'elle envoie la pluie et le soleil, qu'elle fasse pousser une herbe grasse et une récolte abondante, qu'elle épargne aux humains les tremblements de terre et les avalanches, les attaques de loups et d'ours, il faut maintenir une bonne relation avec elle. Et cela se négocie. On part du principe que la Nature est plus forte que l'humain, et on redoute son caractère lunatique. Son territoire, elle le partage avec les humains comme avec toutes les autres espèces, mais une fois sorti de son village et de la communauté qu'il forme avec les autres humains, l'homme n'est plus maître de ce qui l'entoure.

Et c'est justement dans cette Nature non-maîtrisée que le chant a lieu. Le basa ahaide s'imprègne de cet environnement, le chanteur absorbe quelque chose de sa nature sauvage, et il s'y intègre en chantant. D'ailleurs, le mot basa veut dire sauvage, dans le sens d'une non-maîtrise, d'une nondomination de ce milieu par l'homme. Le chant est donc beaucoup plus qu'une ode à la Nature, il est une conversation, une négociation, une demande d'appartenance et de partage. Et le canto con caja de même. La voix se déploie dans les hauteurs solitaires que le berger fréquente avec son troupeau, c'est là où les paroles lui viennent. Plus tard, lors des grandes célébrations dédiées à la Pachamama, les chanteurs témoignent de cette relation et adressent leurs louanges et leurs demandes collectivement à la Terre Mère, dans le but d'assurer la récolte de la nouvelle saison qui nourrira la communauté. L'environnement naturel est plus ou moins personnifié selon les croyances et les traditions locales, le chanteur peut aussi bien s'adresser à une force extérieure indéfinie qu'à une figure fortement anthropomorphe comme la Pachamama.

Dans tous les cas, il faut se familiariser avec cette Nature, ni bonne ni mauvaise, pour pouvoir espérer la connaitre et attirer sa bienveillance. Il faut soigner une relation faite de respect et d'attention, sachant que cette Nature est aussi capable de nous ignorer, de ne pas nous prendre en compte, de favoriser d'autres espèces que l'espèce humaine. Cela reflète l'image que nous propose la 
philosophe Isabelle Stengers ${ }^{5}$ d'une Gaia pour qui les humains n'ont pas la priorité, pour qui les humains sont peut-être même devenus une maladie à éradiquer.

Stengers parle, reprenant Deleuze, du besoin d'un devenir-sensible, processus urgent quand on considère le désastre écologique préparé par l'homme dans son utilisation anthropocentrique de la planète, désastre aujourd'hui capable de nous mener littéralement à la fin du monde. Le basa ahaide comme le canto con caja exprime ce devenir-sensible, ils le poussent à s'aiguiser, à s'affiner. Le chanteur est un être poreux, les frontières tangibles et intangibles qui le séparent de son environnement se dissolvent dans le chant, il reçoit son milieu en détail et de façon immédiate. Pour des peuples qui traditionnellement vivent en grande partie de ce qu'ils élaborent avec la complicité du milieu naturel (même si nous savons que cela cède de plus en plus aux apports de l'industrie), la sensibilité dont parle Stengers est une évidence, une nécessité absolue, et le chant participe à le maintenir, à le soigner.

La place de l'homme au sein de son milieu ainsi que la hiérarchie qu'il instaure, ou pas, entre les espèces, est particulier à chaque cosmovision. Nous commençons à nous rendre compte que le point de vue de l'occident n'est qu'une vision parmi d'autres. Comme le dit Philippe Descola : "la manière dont l'Occident moderne se représente la nature est la chose du monde la moins bien partagée. "6

La culture basque et la culture andine, bien qu'influencées par la culture occidentale moderne, maintiennent toutes les deux leurs racines bien implantées dans un passé qui les distinguent de cette culture dominante. La culture basque, que l'on peut étendre à la culture vasconne qui couvre plus de la moitié de la chaine des Pyrénées, est pré-indoeuropéenne. La culture des peuples du nord-ouest argentin est pré-incaïque. Ces deux cultures sont liées à des cosmovisions traditionnellement centrées autour des figures de la Terre Mère et du Père Soleil, cosmovision encore très active chez les peuples andins et présente sous la forme atténuée de mythes et légendes chez le peuple basque. Elles comprennent une relative égalité entre les espèces, et même entre tous les éléments de la Nature, puisque les pierres, l'eau, le vent, et les phénomènes météorologiques peuvent eux aussi être investis par des forces vivantes. Le chant ancestral est souvent en dialogue avec les autres espèces qui habitent le milieu, et qui sont en quelque sorte des passeurs ou des intermédiaires entre les humains et les entités intangibles de la montagne. Dans le basa ahaide, le chanteur emprunte la voie tracée par les oiseaux de montagne, le chant se déploie selon leur mouvement pour atteindre les sommets. Dans le canto con caja, les humains reprennent le mouvement et la voix des perdrix des Andes qui sortent avant l'arrivée des pluies pour chanter tout en marchant en rond. Les coplas intègrent des sonorités rauques et denses des animaux domestiques et sauvages, ainsi que les sons du vent, de l'eau, et de la roche en mouvement. Ces emprunts, imitations et inspirations sont autant de conversations menées entre l'homme et les autres espèces, et à travers eux entre l'homme et les forces invisibles ou imprévisibles du monde. Si l'homme se pensait supérieur aux autres espèces, s'il pensait dominer complètement son environnement, ces pratiques n'auraient pas de raison d'être. Le chant n'exprime pas la prééminence de l'homme, il sert à maintenir et à soigner une continuité et un équilibre entre les éléments qui composent l'univers, tel que le font également toutes les autres espèces avec leurs propres rites, danses et chants.

5 STENGERS Isabelle, Au temps des catastrophes, Paris, Les empêcheurs de penser en rond/La découverte, 2009. 
Des anthropologues comme Eduardo Viveiros de Castro ou Philippe Descola ont beaucoup contribué à déconstruire l'absolutisme de la pensée occidentale, ouvrant la possibilité de concevoir une logique autre du monde et donc une organisation sociale autre vis-à-vis du milieu naturel, ce qui nous permet aujourd'hui d'imaginer non seulement d'apprendre des sociétés différentes de la société occidentale moderne, mais aussi d'importer des techniques et la pensée des autres peuples au service de notre société, pour y apporter des changements. L'écologie et tout ce qu'elle promeut comme développement durable est un des secteurs qui aurait beaucoup à apprendre des communautés des montagnes dont il est question ici.

Les pratiques de chant ne sont qu'un aspect de la relation d'équilibre que ces communautés de montagne de tradition orale maintiennent avec leur environnement, mais on aurait tort de les considérer comme marginales. La biologiste Donna Haraway souligne l'importance des "histoires qu'on utilise pour raconter d'autres histoires"7. C'est-à-dire que la forme que l'on donne à ce qu'on voudrait transmettre va affecter la manière dont celui à qui on le destine le reçoit. Le chant est un exemple exemplaire de cette attention portée à la forme. Il suscite une réponse chez l'auditeur qui est à la fois physique, émotionnelle et intellectuelle. Il ancre ce qu'il transmet dans le corps de l'autre, il crée, le temps du chant, une continuité entre son corps et le corps de celui qui écoute. Mesuronsnous assez l'effectivité de ce geste? Quelque chose qui nous affecte à ces trois niveaux simultanément a probablement plus de chance d'être assimilé durablement, en nous touchant personnellement. Ce que l'on transmet par le basa ahaide, par le canto con caja, n'est pas une information que l'on possède et qu'on l'on donne, mais une relation que l'on fait comprendre, dans laquelle on invite l'auditeur à entrer. Dans le cas des chants qui nous servent d'exemple dans cet essai, il s'agit une relation à un environnement naturel, tandis que d'autres chants traitent d'autres relations.

La relation créée par le chant reflète la notion du tiers inclus ${ }^{8}$ telle qu'elle est utilisée par Brian Massumi dans sa description de la pensée du vivant ${ }^{9}$. Le chanteur de basa ahaide et l'oiseau qu'il suit par son chant existent chacun séparément, mais dans la rencontre que permet le chant, l'élément humain et l'élément oiseau (mais aussi l'élément humain et l'air, la roche, la forêt) vont se rencontrer dans une zone d'inclusion mutuelle, où ils se mélangent sans fusionner, et dans laquelle ils vont pouvoir expérimenter tout ce qui pourrait naître de leur composants réunis. Cela génère un potentiel qui excède ce que chacun est, et dans lequel chacun puisera un développement possible. Ils créent ensemble du conditionnel, un "ce que je pourrais" à partir d'un "ce que je peux", et dont une partie va s'actualiser. Le chanteur joue en quelque sorte à être oiseau, mais comme le souligne Massumi, il ne s'agit en aucun cas d'une imitation. Le devenir-animal du chanteur emprunte les « tensions » et «tendances» proposées par son partenaire, il est orienté, dans le cas du basa ahaide, par la « rapacitude » plutôt que par le rapace, ce qui permet d'amener le rapace dans des situations où il

HARAWAY Donna, Staying with the trouble, making kin in the Chthulucene, Londres, Duke University Press, 2016.

8 notion issue d'une réflexion sur la physique quantique par Stéphane Lupasco : LUPASCO Stéphane, Le principe d'antagonisme et la logique de l'énergie - Prolégomènes à une science de la contradiction, Paris, Éditions du Rocher, 1987. 
n'arriverait jamais par lui même, propulsée par «l'enthousiasme du corps » du chanteur en dehors de ses limites normales. ${ }^{10}$

Cet excédent est la partie créative qui définit le style, qui lui définit une direction pour le développement. Le chanteur connait la mélodie qu'il va chanter, c'est "ce qu'il peut". Mais la façon dont il va l'interpréter et qui va être sa marque, son style, son innovation par rapport à ce qu'il sait, va naître de ce que l'autre, l'oiseau, l'air, la roche, la forêt, permet à son corps de projeter. Ce sont les éléments du contexte, ou pour reprendre la précision de Massumi, de la situation, qui donnent au corps du chanteur l'élan nécessaire à un dépassement de soi.

En décrivant cette relation, Massumi met le doigt sur comment le corps pense, sur la capacité du corps à réfléchir. Le conditionnel, le "ce que je pourrais", est de fait une abstraction, une projection dans l'avenir, et dans le chant, comme dans toute interaction, cette abstraction est immanente, elle se fait de façon immédiate, simultanément au geste, par le geste et non pas par rapport au geste.

Massumi pousse cette réflexion jusqu'à ses conséquences politiques, proposant une politique animale, une politique du vivant, qui valorise l'actualisation de cette partie de "ce que je pourrais" capable de faire évoluer "ce que je peux". En politique, ce sont les règles établies par la macropolitique qui doivent céder leur prééminence aux innovations spontanées de la micro-politique, innovations qui répondent à ce qu'une situation spécifique fait naître à partir de sa dynamique propre. Quand Naess parle de réactions spontanées, le "non !" ou le "oui !" qui surgit instinctivement, avant toute réflexion cognitive ${ }^{11}$, il parle de cet élan du corps qui dépasse le "normal", la simple exécution, pour prendre une direction. C'est ce que Massumi a appelé « l'esquéité », le surnormal, cette chose qui dépasse le prévu pour dessiner un à-venir, qui définit un style, une orientation.

Le chant permet d'enclencher ce dépassement de soi et d'en prendre conscience, et surtout, de prendre plaisir à se laisser emporter dans un mouvement qui n'est qu'en partie prévu, qu'en partie maîtrisé. Cette capacité de saisir, de suivre et de réagir dans l'immédiat, de s'ouvrir intégralement à une situation pour qu'elle devienne un tremplin vers l'innovation, serait clairement enrichissante pour d'autres domaines que le chant. Et de fait, ce sont des processus qui opèrent dans toutes les situations de la vie, auquels nous ne prêtons peut-être pas assez d'attention.

Quand on regarde la situation actuelle de la planète et l'impasse dans laquelle la consommation excessive typique des pays développés est en train de nous mener, on peut commencer peut-être à comprendre l'importance que pourrait avoir cette forme spécifique de compréhension que nous propose le chant. Le chant n'est pas le lieu de décisions vis-à-vis d'une politique environnementale, mais il participe à poser les bases : la possibilité de ressentir et avoir confiance en la relation que nous voudrions entretenir avec notre milieu, ou plus précisément, avec notre lieu. Et c'est à partir de cette relation qu'on peut formuler nos convictions et les défendre, qu'on peut développer les arguments qui initient et justifient nos actions.

Le chant peut aussi, peut-être, nous permettre de voir qu'il n'y a pas une unique vision du monde qui représenterait la vérité, et que même les choses qui nous semblent les plus acquises ne résistent pas à la déconstruction que peuvent opérer d'autres cosmovisions. Il n'est pas question d'abandonner

\footnotetext{
10 Massumi reprend et développe le devenir-animal tel qu'il est conçu par DELEUZE Gilles, GUATTARI Félix, Mille Plateaux, Paris, Éditions de Minuit, 1980.
} 
nos perspectives pour adopter celles d'un autre peuple, mais d'ouvrir notre culture aux enseignements venus de ceux qui ne voient pas le monde comme nous. Parce que nous ne le disons pas assez, ce que nous décidons ou acceptons de croire a des effets concrets sur nous, sur notre vie, sur les relations que nous entretenons avec les autres et avec notre milieu. Et ce sont ces effets qui petit à petit vont nous amener à formuler notre vérité. Cela nous donne un grand pouvoir pour contrer le récit dominant qui voudrait que l'on n'ait pas le choix, qu'il n'y a pas d'alternative. Les communautés montagnardes des Pyrénées et des Andes nous ouvrent déjà des perspectives enrichissantes, et cela ne peut que nous encourager à poursuivre nos recherches en direction d'autres cultures, dans d'autres parties du monde, pour continuer à étendre notre capacité à percevoir, pour continuer à développer notre devenir-sensible. 